\title{
Surface Modification of Teflonated Carbon Fabric by Ultrasound-Assisted Radiation Induced Grafting Copolymerization
}

\author{
Nur Afifah Zubair ${ }^{1,2}$, Mohamed Mahmoud Nasef ${ }^{4 *}$, Ting Teo Ming ${ }^{5}$, Ebrahim Abouzari-Lotf ${ }^{2,3}$, Ezzat Chan \\ Abdullah $^{1}$ \\ ${ }^{1}$ Malaysia-Japan International Institute of Technology, Universiti Teknologi Malaysia, Jalan Sultan Yahya Petra, \\ 54100 Kuala Lumpur, Malaysia \\ ${ }^{2}$ Center of Hydrogen Energy, Institute of Future Energy, Universiti Teknologi Malaysia, Jalan Sultan Yahya \\ Petra, 54100 Kuala Lumpur, Malaysia \\ ${ }^{3}$ Department of Chemical Engineering, School of Chemical and Energy Engineering, Faculty of Engineering, \\ Universiti Teknologi Malaysia, 81310 Johor Bahru, Johor, Malaysia \\ ${ }^{4}$ Department of Chemical Engineering, Faculty of Engineering, Universiti Teknologi Petronas, Bandar Seri \\ Iskandar, 31750 Tronoh, Perak, Malaysia \\ ${ }^{5}$ Radiation Processing Technology Division, Malaysian Nuclear Agency, 43000 Kajang, Selangor, Malaysia
}

*Corresponding author: mohamed.nasef@utp.edu.my

Article History

Received: March 10, 2018 Received in revised form: Novenber 16, 2018 Accepted: December 3, 2018 Published Online: February 10, 2019

\begin{abstract}
A simple and effective modification method was carried out to improve the surface properties of teflon-impregnated carbon fabric (TICF) by radiation induced grafting (RIG) of glycidyl methacrylate (GMA). The grafting reaction was carried out using an ultrasound-assisted technique in comparison with a conventional grafting technique. The degree of grafting was investigated at various reaction times for both systems. The grafting efficiency for ultrasonic-aided technique reached $85 \%$ compared to $53 \%$ in conventional grafting technique. This significant improvement is probably due to the emergence of monomers diffusion on the grafting sites caused by ultrasonically assisted grafting reaction. Scanning electron microscopy (SEM) and Fourier transform infrared (FTIR) spectroscopy have provided evidences for incorporation of poly(GMA) grafts. The grafting under ultrasonic condition was proven to be more efficient and economical as indicated by the increase in DG and reduction in the homopolymer contamination accompanied by homogenous grafting and smoother surfaces in TICF.
\end{abstract}

Keywords: Radiation induced grafting, carbon fabric, ultrasound, surface modification

\subsection{INTRODUCTION}

The carbon fibers are a class of porous materials with an effective specific surface area and narrow pore size distribution [1] which exhibit excellent chemical and thermal stabilities [2]. It is an ideal material for many applications such as adsorption and separation [3], catalysis [4] and fuel cell applications [5]. However, carbon fabric surface is soft, smooth and inert, thus the degree of surface interaction between the fibers and matrix is rather poor. Hence, excellent properties of the material cannot be deployed. Surface modification considering the roughness and introduction of suitable functional groups can enhance the chemical bond and physical interactions between fibers and allow further modifications.

Various modification methods have been reported for improving the surface properties of carbon fabric including thermal treatment [6], electrochemical oxidation [7], plasma treatment [8], gas-phase oxidation [9], coating treatment [10] and irradiation treatment [11]. Modification of fibers by radiation induced grafting (RIG) with high-energy is a fascinating method for imparting various functional polymeric materials for various range of applications [12]. Thus, RIG has been extensively 
explored as a modest technique to alter surface properties of polymeric materials, such as films, fibers, powders and moulded objects $[13,14]$.

Few methods were introduced to enhance the grafting efficiency and reduce monomer consumption. This includes grafting in an emulsion medium, combining grafting with RAFT and using single step techniques involving functionalized monomer [15]. Recently, ultrasound was reported to synergize chemical reactions through product enhancement, reaction time saving, solvent consumption reduction and extensive energy saving [16]. Recently, Nasef et al. [17] has reported an synergetic RIG for 4-vinylpyridine/glycidyl methacrylate mixtures onto poly(ethylene-co-tetrafluoroethylene) films using ultrasound-aided technique for the first time. The process was proven to be highly effective not only in time shortening and reduction of monomer consumption but also in grafting product enhancement, surfaces smoothing and eliminating of contamination from homopolymer compared to grafted films obtained from conventional grafting.

Nevertheless, little attention has been given to the modification of carbon fibers by RIG using ultrasound assisted technique. Applying such techniques is likely to overcome the grafting difficulty caused by its graphitized structure of carbon and limited counts for forming reactive sites of the carbon fiber [18]. The present study is an attempt to graft glycidyl methacrylate (GMA) onto teflon-impregnated carbon fabric (TICF) using RIG technique assisted with ultrasonic-aided technique. In this study, GMA was selected as grafted monomer, which attracted high interest due to its epoxy group that can be modified into various functional groups. The epoxy ring can be opened under mild reaction conditions offering the possibility for introducing a variety of ionic moieties suitable for various applications [19]. The grafting substrates were mainly characterized by means of scanning electron microscopy (SEM) and Fourier transform infrared (FTIR).

\subsection{METHODOLOGY}

\subsection{Materials}

Teflon-impregnated carbon fabric (TICF) with thickness of $0.65 \mathrm{~mm}$ was obtained from E-TEK, Inc. USA. Glycidyl methacrylate (GMA) was obtained from Sigma Aldrich and used without any further purification. Methanol and ethanol was reagent grade and used as received.

\subsection{Irradiation of Teflon-Impregnated Carbon Fabric}

TICF were rinsed using ethanol for few times and dried in a vacuum. The irradiation of the substrates were performes by by an EB accelerator (EPS 3000, Nissin High Voltage, Japan) to a total dose of 300 at $25 \mathrm{kGy}$ per pass under $\mathrm{N}_{2}$ atmosphere. The accelerating voltage was maintained at $2 \mathrm{Mev}$ and at a beam current of $10 \mathrm{~mA}$ at ambient temperature. Dry iced was used to stabilize the radicals formed in the substrates during irradiation of high absorbed dose. The TICF were kept in a thermally sealed polyethylene bag to remove any moisture interference and the irradiated substrates were immediately stored in an ultracool freezer at $-60{ }^{\circ} \mathrm{C}$ prior to use.

\subsection{Graft Polymerization}

Grafting reaction of GMA onto TICF was carried out using a grafting apparatus. The grafting procedure started with placing the irradiated substrates in a glass ampoule, which was tightly sealed and evacuated. A $\mathrm{N}_{2}$-bubbled monomer solution composed of GMA/methanol with a concentration of 20 vol\% was transferred to the ampoule through a special vacuum stopcock. The reaction ampoule was placed in a preheated ultrasonic waterbath with ultrasonic energy $(40 \mathrm{kHz}, 300 \mathrm{~W})$. The reaction was performed to a desired range of reaction times while maintaining the temperature at $60{ }^{\circ} \mathrm{C}$. After the reaction completed, the grafted TICF was removed and cleaned from the unreacted monomer or any homopolymerised monomer using ethanol. The grafted carbon fabric was dried under vacuum at $50{ }^{\circ} \mathrm{C}$ for $24 \mathrm{~h}$ until the constant weight was obtained. The sample was removed and kept under a desiccator over fresh silica gel for 1 hour before weighing. The degree of grafting (DG) was obtained using equation 1 with the reference to the content of grafted carbon fibers.

$$
D G(\%)=\frac{W_{1}-W_{0}}{W_{0}} \times 100 \%
$$

where $W_{0}$ and $W_{1}$ are the weights of carbon substrates before and after grafting respectively. The grafting efficiency [13] is calculated from equation 2 : 


$$
G E(\%)=\frac{W_{1}-W_{0}}{W_{2}-W_{0}} \times 100 \%
$$

where $W_{2}$ is the weight of the grafted sample before removal of homopolymer.

For comparison purpose, the conventional grafting reaction was carried out under similar grafting condition by placing the reaction ampoule into a thermostatic water bath at temperature of $60^{\circ} \mathrm{C}$. The DG and GE were also determined using equations 1 and 2.

\subsection{Characterization Of Grafted Ticf}

A Perkin Elmer-Spectrometer FTIR 2000 Explorer was used to characterise the chemical structures of pristine and grafted TICF. The measurement was carried out in a transmission mode in the wavenumber range of $4000-400 \mathrm{~cm}^{-1}$ at a resolution of $4 \mathrm{~cm}^{-1}$. The surface morphology and roughness of TICF were observed by SEM analysis using FEI Quanta 4000 microscope with an acceleration voltage of $2 \mathrm{kV}$ and magnification of 500x.

\subsection{RESULTS AND DISCUSSION}

The effects of various reaction time on the DG of GMA onto TICF with and without ultrasound assistance were investigated as shown in Figure 1a. Notably, the DG in the ultrasound-aided technique was higher than conventional technique despite having similar increasing trend in both techniques with the rise in reaction time. Moreover, grafting efficiency [13] was greatly boosted by applying ultrasound to grafting system. For instance, the GE was jumped from 53\% to $85 \%$ after $4 \mathrm{~h}$ when ultrasound was applied. The remarkable increase can be attributed to the increase in the monomer diffusion to grafting sites and also caused by the upsurge of the activate radical arising from the heat associated with sonication. This phenomenon indicates that the ultrasound-assisted could enhance the kinetics grafting of GMA onto carbon fiber substrates. The implosions of ultrasound generate cavitation bubbles and the supplemented heat improving the rate of grafting reaction. Subsequently, the radicals' activation increases thus intensified the collision of monomer molecules leading to noticeable enhancement in GE and eventually reduction in the monomer consumption. These findings are in good agreement with previous reported literature [17].

(a)

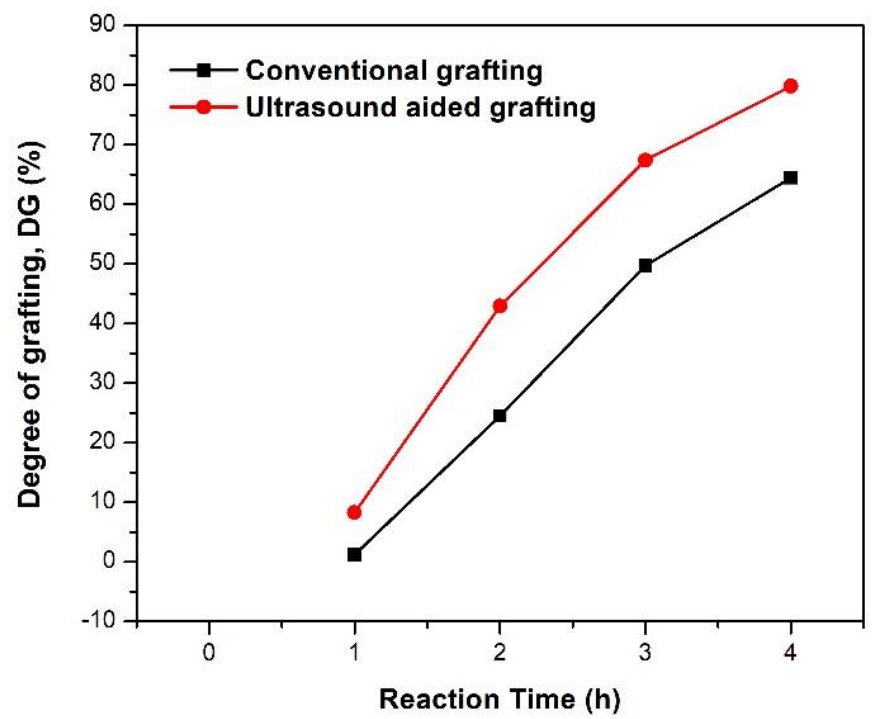

(b)

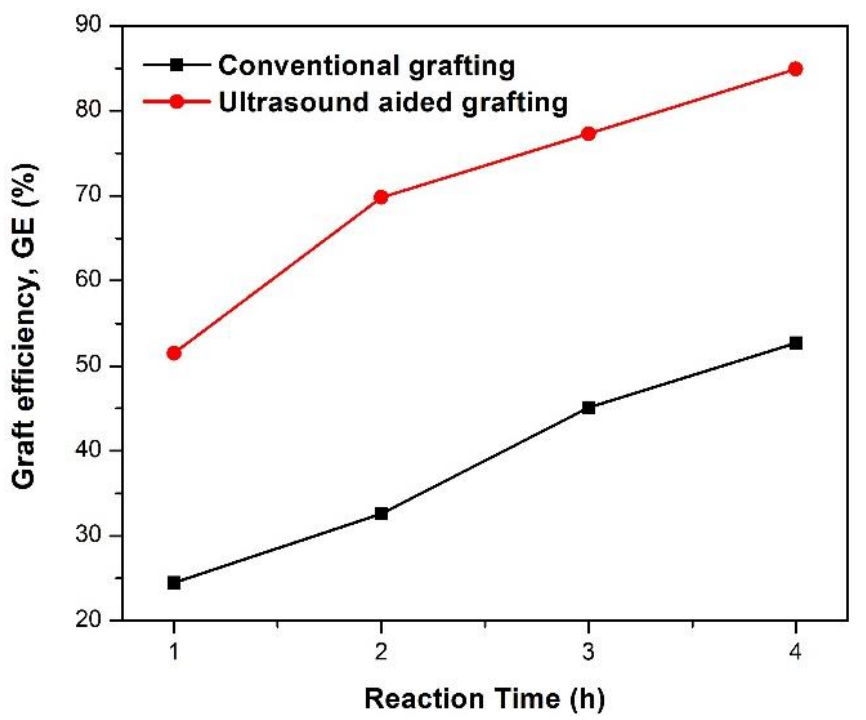

Figure 1. Variation degree of grafting and grafting efficiency. Grafting conditions are $300 \mathrm{kGy}$ of adsorbed dose, $20 \%$ monomer concentration and $60^{\circ} \mathrm{C}$ temperature. 
The variation of grafting efficiency with time for grafting of GMA onto TICF using conventional and ultrasound-aided grafting techniques is shown in Figure 1b. The ultrasound assisted grafting reaction exhibited higher GE and monomer conversion than conventional grafting. The ultrasound-aided grafting exhibited more than 50\% GE compared to conventional grafting at the same condition reaction. This observation confirms that the ultrasound technique encouraged effective monomer diffusion and subsequent rise in its supply to the grafting sites caused by exclusion of homopolymer chains from the film surfaces through implosion of cavitation bubbles. Thus the implosion of bubbles near a surface coincides with arising in the number of activated radicals by the associated heat resulting from sonication. Therefore, the active radical involved in initiation with the growing of monomer molecules leading to higher GE up to $85 \%$ in the ultrasound assisted method. This significant improvement of $24 \%$ is most probably due to the enhancement of monomers diffusion to grafting sites caused by ultrasonically assisted grafting reaction. Additionally, the TIGF produce by ultrasound-aided grafting exhibited 52\% GE within $1 \mathrm{~h}$ as compared to conventional grafting which required $4 \mathrm{~h}$ to obtain $53 \%$ of grafting efficiency. It was clearly proved that the grafting reaction using ultrasound has advantage in shortening the reaction time and energy saving to yield high grafting efficiency.

Figure 2 shows the SEM images of TICF before and after grafting with GMA. Clearly, the surface of pristine TICF seems to be relatively smooth as shown in Figure 2a. However, there is a coating layer of teflon on the surface of carbon fiber which keep the fibrous structure stick to each other. On the other hand, grafting of GMA on the TICF made their surface rougher and groovier suggesting penetration of poly (GMA) graft to thin teflon layer and reaching the surface of carbon fibers. Moreover, the size of carbon fibers obtained under conventional and ultrasonic conditions was slightly increased compared to pristine TICF (Fig. 2b and 2c). The surface of TICF-g-PGMA obtained from conventional grafting (Fig. 2b) is rather rougher and irregular compared to the ultrasound-aided grafted fibers (Fig. 2c), which interestingly displayed homogeneous and smoother surfaces in the grafted fibers. The roughness of the TICF surface was improve by EB irradiation graft which is highly important in some applications [18]. This observation indicates that ultrasound-assisted grafting imparts better and smoother surface properties than conventional grafting of the carbon substrates.
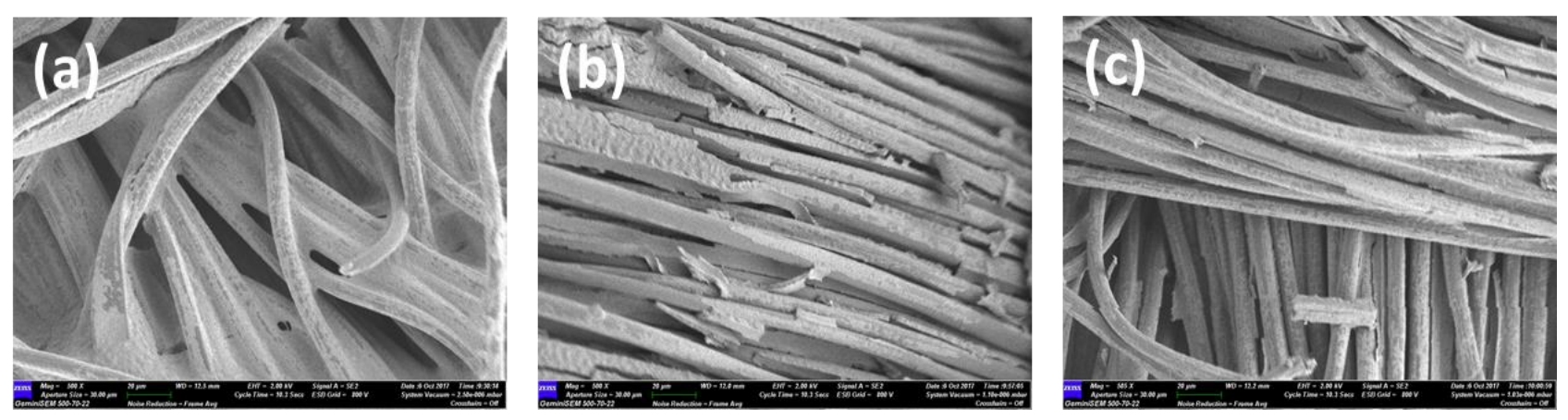

Figure 2. SEM images of (a) pristine teflon-impregnated carbon fibers and TICF- $g$-PGMA obtained under (b) conventional and (c) ultrasonic conditions.

Figure 3 presented FTIR spectra of poly (GMA) grafted carbon fibers obtained by conventional and ultrasound assisted grafting compared to pristine carbon fibers. The adsorption band at $1240 \mathrm{~cm}^{-1}$ observed for the pristine and modified carbon fibers is attributed to the $\mathrm{C}-\mathrm{C}$ stretching band. The grafted fibers from both grafting techniques showed an absorption peak at $850 \mathrm{~cm}^{-1}$, representing the stretching vibration of the epoxy group originated from the incorporated poly (GMA). This result is supported by the appearance of strong peaks at $1720 \mathrm{~cm}^{-1}$ which is assigned the -CO in the methacrylate (ester) group, confirming the presence of poly (GMA) in the grafted TICF. However, the characteristic band at $1720 \mathrm{~cm}^{-1}$ for the TICF- $g$-PGMA from conventional grafting are more intense compared to conventional grafting under the same reaction conditions. This might be due to the presence of homopolymer chain of GMA and inhomogenous grafting of GMA on the TICF surfaces as can be seen in Figure 2b. Thus, the FTIR features provide an evidence for the successful grafting of the GMA onto the TICF, and also the enhancement of GE using ultrasound-assisted grafting technique. 


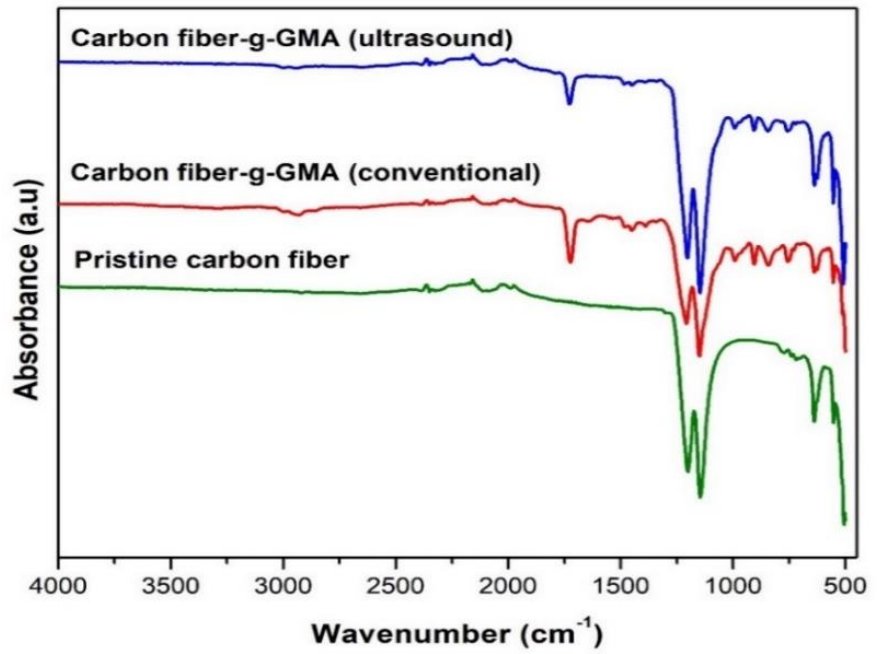

Figure 3. FTIR spectra of pristine teflon-impregnated carbon fibers and TICF- $g$-PGMA obtained under conventional and ultrasonic conditions.

\subsection{CONCLUSION}

The surface modification of teflonated carbon fabric was successfully performed using radiation induced grafting copolymerisation. The RIG of GMA onto TIGC was intensified by conducting the reaction under ultrasound aid. Notably, the application of ultrasound prompted higher degree of grafting and grafting efficiency compared to conventional grafting method. FTIR analysis confirmed that the sample obtained by applying ultrasound presented higher DG under same reaction parameters compared to conventional grafting. The grafted carbon fibers prepared from ultrasound aided grafting were found to have better surface properties compared to conventional grafting as displayed by SEM analysis. This finding suggested that the grafting system with ultrasound-assisted techniques has advantages in time consumption and extensive amount of energy saving.

\section{Acknowledgements}

This work was funded by Universiti Teknologi Malaysia (UTM) and Malaysia Thailand Joint Association (MTJA) program (Vot number 4C116).

\section{References}

[1] Zhang, L., et al. 2013. Controlling the Effective Surface Area and Pore Size Distribution of sp2 Carbon Materials and Their Impact on the Capacitance Performance of These Materials. Journal of the American Chemical Society. 135(15): 5921-5929.

[2] Jin, L., et al. 2016. Improvement of interfacial strength and thermal stability of carbon fiber composites by directly grafting unique particles: functionalized mesoporous silicas. RSC Advances. 6(84): 80485-80492.

[3] Burchell, T.D. and R.R. Judkins, 1997. A Novel Carbon Fiber Based Material And Separation Technology. Energy Conversion and Management. 38: S99-S104.

[4] Yang, S., et al. 2015. .Activated Carbon Fiber As Heterogeneous Catalyst Of Peroxymonosulfate Activation For Efficient Degradation Of Acid Orange 7 In Aqueous Solution. Separation and Purification Technology. 143: 19-26.

[5] Zhang, X. and Z. Shen. 2002. Carbon Fiber Paper For Fuel Cell Electrode. Fuel. 81(17): 2199-2201.

[6] Stepashkin, A.A., et al. 2014. Surface Treatment Of Carbon Fibers-Fillers For Polymer Matrixes. Inorganic Materials: Applied Research. 5(1): 2227.

[7] Liu, J., et al., 2010. Interfacial And Mechanical Properties Of Carbon Fibers Modified By Electrochemical Oxidation In $\left(\mathrm{NH}_{4} \mathrm{HCO}_{3}\right) /\left(\mathrm{NH}_{4}\right)_{2} \mathrm{C}_{2} \mathrm{O}_{4} \cdot \mathrm{H}_{2} \mathrm{O}$ Aqueous Compound Solution. Applied Surface Science. 256(21): 6199-6204.

[8] Chien, H.-H., et al. 2018. .Improved Performance Of Polyaniline/Reduced-Graphene-Oxide Supercapacitor Using Atmospheric-Pressure-Plasma-Jet Surface Treatment Of Carbon Cloth. Electrochimica Acta., 260: 391-399. 
[9] Zhang, X., et al., 2017. Vapour-Phase Hydrothermal Synthesis Of $\mathrm{Ni}_{2} \mathrm{P}$ Nanocrystallines On Carbon Fiber Cloth For High-Efficiency $\mathrm{H}_{2}$ Production And Simultaneous Urea Decomposition. Electrochimica Acta. 254: 44-49.

[10] Kariminejad, A., et al. 2016. Effects Of PACVD Parameters Including Pulsed Direct Current And Deposition Time On Nanostructured Carbon Coating Deposited On Carbon Fiber Fabrics. Materials \& Design. 106: 184-194.

[11] Andrianova, N.N., et al. 2017. Modification Of Polyacrylonitrile Carbon Fibers By Highfluence Ion Irradiation. Journal of Physics: Conference Series, . 941(1): 012028.

[12] Chen, J., et al. 2007. Polymer Electrolyte Hybrid Membranes Prepared By Radiation Grafting Of P-Styryltrimethoxysilane Into Poly(Ethylene-CoTetrafluoroethylene) Films. Journal of Membrane Science. 296(1): 77-82.

[13] Shen, L., et al. 2017. Surface modification of polyvinylidene fluoride (PVDF) membrane via radiation grafting: novel mechanisms underlying the interesting enhanced membrane performance. Scientific Reports. 7(1): 2721.

[14] Shin, I.H., et al. 2017. Surface Modification Of PVDF Membrane By Radiation-Induced Graft Polymerization For Novel Membrane Bioreactor. Journal of Industrial and Engineering Chemistry,. 46: 103-110.

[15] Nasef, M.M., et al. 2014. .Advances In The Development Of Functional Polymers Using Radiation Induced Emulsion Polymerization. Recent Res Dev Polym Sci. 12: 107-128.

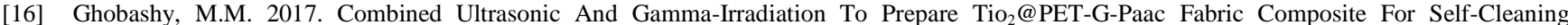
Application. Ultrasonics Sonochemistry,. 37(Supplement C): 529-535.

[17] Nasef, M.M., et al. 2017. .Intensifying Radiation Induced Grafting Of 4-Vinylpyridine/Glycidyl Methacrylate Mixtures Onto Poly(Ethylene-CoTetrafluoroethylene) Films Using Ultrasound. Radiation Physics and Chemistry. 134: 56-61.

[18] Zhang, S., et al. 2009. Irradiation-Induced Grafting Of Acrylonitrile Onto Activated Carbon Fiber. Polymers for Advanced Technologies. 20(12): $1168-1173$

[19] Moawia, R.M., et al. 2016. Modification Of Flax Fibres By Radiation Induced Emulsion Graft Copolymerization Of Glycidyl Methacrylate. Radiation Physics and Chemistry. 122: 35-42. 